\title{
An enhanced technique for strongly nonlinear oscillators with a harmonic restoring force
}

\author{
Yusry El-Dib ${ }^{1}$ \\ ${ }^{1}$ Ain Shams University
}

May 5, 2020

\begin{abstract}
An enhanced analytical technique for nonlinear oscillators having a harmonic restoring force is proposed. The approach is passed on the change of the auxiliary operator by another suitable one leads to obtain a periodic solution. The fundamental idea of the new approach is based on obtaining an alternative equation free of the harmonic restoring forces. This method is a modification of the homotopy perturbation method. The approach allows not only an actual periodic solution, but also the frequency of the problem as a function of the amplitude of oscillation. Three nonlinear oscillators including restoring force, the simple pendulum motion, the cubic Duffing oscillator, the Sine-Gordon equation are offered to clarify the effectiveness and usefulness of the proposed technique. This approach allows an effective mathematical approach to noise and uncertain properties of nonlinear vibrations arising in physics and engineering.
\end{abstract}

\section{Hosted file}

Resroring Force 95.pdf available at https://authorea.com/users/303600/articles/433786-anenhanced-technique-for-strongly-nonlinear-oscillators-with-a-harmonic-restoring-force 